\title{
INTENSITY OF SATELLITE RADAR-ALTIMETER RETURN POWER OVER CONTINENTAL ICE: A POTENTIAL MEASUREMENT OF KATABATIC WIND INTENSITY
}

\author{
By F. Remy, C. Brossier, and J.F. Minster \\ (UM 39, Groupe de Recherche de Géodésie Spatiale, 18 avenue Edouard-Belin, \\ 31055 Toulouse Cedex, France)
}

\begin{abstract}
We analyse, above continental ice, the various factors which affect the power return of the Seasat radar altimeter as measured by its Automatic Gain Control (AGC). Corrections of effects due to the AGC loop control are first applied. AGC is then normalized by positioning the half-power point at the middle of the instrument receiving window. This operation is valid for both surface and volume scattering. Over a part of Antarctica between long. $90^{\circ}$ and $150^{\circ} \mathrm{E}$., the remaining variations of AGC are of the order of $15 \mathrm{~dB}$. Most of these variations occur on a large scale $(>100 \mathrm{~km})$ and are correlated with the katabatic wind intensity. This indicates that AGC measures either surface roughness of the ice, which is related to wind intensity, or grain-size which could also be dependent on the wind. In-situ measurements support the evidence that the radar altimeter is more sensitive to surface scattering. These data could therefore provide a measurement of the intensity of katabatic winds over the continental ice.
\end{abstract}

\section{INTRODUCTION}

A satellite radar altimeter transmits pulses towards the sub-satellite point and receives a returned signal which is affected by its reflection at the surface and/or the scattering by the sub-surface. The main information is the time for the pulse to be returned, which gives the height between the surface and the reference ellipsoid. Thus, the primary interest of these measurements is the construction of a very precise surface topography (see for continental ice sheets, Zwally and others, 1983; McIntyre and Drewry, 1984). Remy and others (1989) showed that an adapted inverse technique may provide a topography with a sub-metric precision, even with the Seasat data. The altimeter of the future ERS-1 European satellite will provide such a topography of continental ice up to lat. $82^{\circ} \mathrm{S}$.

However, altimeter data also include other parameters, which are still little explored. Not only could their physical interpretation be useful for other glaciological topics, but also they can provide information about the physics of the altimetric measurements.

Indeed, Ridley and Partington (1988) suggested, using average Seasat altimeter wave forms from three small regions of Antarctica, that both volume and surface scatterings affect the signal to variable extents. Recently, Partington and others (1989) further argued, using average Seasat and Geosat wave forms, that for dry-snow regions, volume scattering dominates the altimetric return. These results are in opposition to previous theoretical and empirical studies which indicate that near-vertical radar returns over dry snow are dominated by surface scattering at the frequency used for radar altimeters (Fung and Eom, 1982; Matzler, 1987). An empirical study of the radar wave forms is very difficult. As a matter of fact, if the signal is a surface signal, the wave forms are affected both by the pointing angle of the altimeter and by the surface slope: both effects cannot be separated and both parameters are poorly known. The wave forms are also affected by the large-scale undulation curvature (Martin and others, 1983) and by the medium-scale features: snow dunes or sastrugi. If the signal is affected by volume scattering, the shape of the wave forms also depends on the dielectric properties of the sub-surface and on the size and density of the scattering particles, both effects also being inseparable (Ridley and Partington, 1988). Too many parameters affect the radar wave forms to allow very firm conclusions on the relative importance of the various effects.

Using Seasat data, the aim of this paper is to study the total energy returned by the surface to the satellite, over continental ice, both to understand better the physics of the scattering, and to interpret the geophysical aspect of the signal. Indeed, we will show that the variations of the intensity of the signal are very large on large distance scales and that only a few parameters can affect it significantly. Operational algorithms used for the estimation of the parameters of the "Geophysical Data Records" and for the control loop of the instrument were designed for the ocean surface. Thus, the return power, as well as the other parameters, first has to be re-estimated directly from the returned pulse wave form.

\section{DEFINITION AND CORRECTION}

\section{a. AGC loop correction}

The design of the Seasat altimeter has been given by McArthur (1978). The return wave form of the radar pulse can be seen as the histogram of the return energy, as sampled by a series of 60 gates. Each gate measures the energy during $3.125 \mathrm{~ns}$. The energy return is controlled by an "Automatic Gain Control (AGC) tracking loop" which acts on the transmitted energy intensity, so that the total return energy is maintained as nearly constant: the system estimates the total energy $E$ of the return signal and compares it with a reference level $E_{\mathrm{r}}$. If $E$ is larger than $E_{\mathrm{r}}$, the next pulse will be more attenuated, and conversely.

To smooth the AGC sequence and avoid white noise above the ocean, only $1 / 16$ of the correction is applied. Thus, if $E$ and AGC are compared for successive measurements (Fig. 1), it can be verified that $E$ varies around the reference value, depending on the variation of AGC. In the case of the ocean surface, the lag between variations of AGC and variation of $E$ is extremely. short. On the contrary, in the case of ice sheets, sea ice, or sand land, the AGC loop only readjusts on time-scales of seconds.

To correct for the AGC loop inefficiency, one has to redefine the AGC by keeping constant the total energy of the retracked wave form. We therefore introduce $A G C^{\prime}$ as:

$$
E_{\mathrm{r}} \times 10^{-\mathrm{AGC}^{\prime} / 10}=E \times 10^{-\mathrm{AGC} / 10}
$$

or

$$
\mathrm{AGC}^{\prime}=\mathrm{AGC}-10 \times \ln \left(E / E_{\mathrm{r}}\right) .
$$



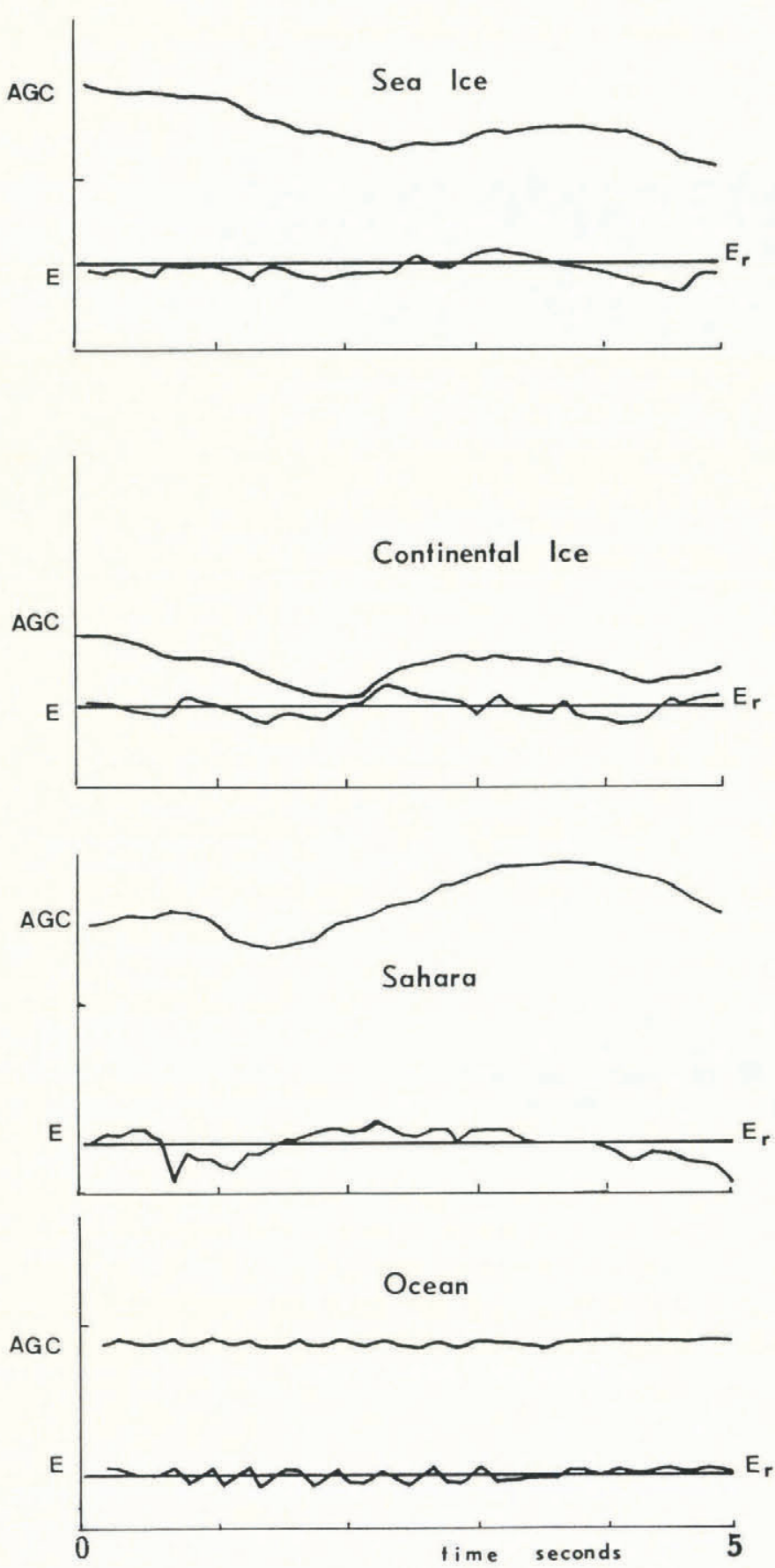

Fig. 1. Evolution of the Automatic Gain Control (AGC) and of $E$ the total energy return above sea and continental ice, Sahara, and the ocean during a $5 \mathrm{~s}$ interval. The mark on the $A G C$ scale of each diagram is the $30 \mathrm{~dB}$ level. The vertical axes have different scales.

The correction to apply to the AGC for estimating the actual energy return is of the order of $2 \mathrm{~dB}$ r.m.s.

\section{b. Tracking-system correction}

As for AGC, the altimetric height is followed with a height-tracking loop, which takes into account only a small part of the topography: the tracking-loop response is too slow for the strong variations of the ice-sheet topography, and the signal is shifted within the receiving window.

This mispositioning of the return pulse in the receiving window also affects the AGC. For example, if the signal is early with respect to the middle of the pre-positioned receiving window, the system will sum up the last gates in excess, $E$ will be too large, and the next AGC value will be adjusted to a larger value. This effect is the same as that introduced by the sea-state bias on the back-scatter coefficient used for measuring wind speeds above the ocean (e.g. Chelton and Wentz, 1986). A five-gates drift of the tracker introduces a $0.7 \mathrm{~dB}$ shift on AGC. This is illustrated in Figure 2, which represents a continuous series of altimeter wave forms above Antarctica. In the case of wave forms Nos 10 to 18 , it is evidence that the AGC variations are only artefacts, due to the mispositioning of the window with respect to the signal.

The operation for correcting this mispositioning is named retracking. In general, the existing retracking algorithms are designed for surface scattering. Martin and others (1983) fitted a theoretical wave form to the 60 gates. This method is very precise but arduous. Brooks and others (1978) assumed that the energy at the middle of the front edge of the wave form is the half value of the maximum power: this method is very easy, but noisy. More recently, Wingham and others (1986) approximated the wave form by a rectangle, chosen to have its centre of gravity and area equal to those of the wave form.

Here, we will use the on-board tracking algorithm: McArthur (1978) showed that the half-power point on the leading edge of the return pulse is equal to the integral of the total return scaled by $53 / 60$, to compensate for the effect of the antenna diagram (this gate number is currently named the AGC gate number). Then, we have to find the gate the energy of which verifies:

$$
P_{\mathrm{r}}(0)=1 / 53 \sum_{-30}^{+30} P_{\mathrm{r}}(\tau)
$$

where $P_{\mathrm{r}}(\tau)$ is the return power of gate $\tau$.

We will see in section 4 that this retracking technique is quite adaptable for surface scattering, and introduces only a sub-decibel error in the case of pure volume scattering.

As the signal is shifted, the calculated sum is not the "true" one; an iterative scheme must be used. A residual drift of one gate, or a height error of $47 \mathrm{~cm}$, leads to a factor error of $(1 \pm 1 / 30)$ on the total return energy, which means a $0.14 \mathrm{~dB}$ error on AGC. Like the method of Wingham and others (1986), this retracking technique uses all range bins to estimate the retracking position, and the noise on the altimeter-return power is smoothed. This method is very easy to implement and gives direct access to the total energy. Both corrections on AGC (AGC loop and retracking) have been applied to a sector of Antarctica, to a sub-set of Seasat data (Fig. 3). In Figure 4, these AGC' values are displayed against AGC for 5000 pulses. The r.m.s of the correction is about $2 \mathrm{~dB}$.

\section{OBSERVATIONS OF AGC FROM SEASAT DATA}

\subsection{Observations along profiles}

The data from Figure 3 have been analysed for AGC Figure $5 \mathrm{a}$ represents the retracked altimetric height, along a $100 \mathrm{~km}$ long path of the Bermuda repeat orbit. The profile shows a large-scale parabolic trend fitted with a polynomial function. $30 \mathrm{~km}$ wavelength undulations are superimposed. The altimetric height is reproducible within $50 \mathrm{~cm}$ r.m.s., which gives confidence to the retracking technique. The corresponding noise on AGC would be about $0.24 \mathrm{~dB}$ r.m.s. Figure $5 \mathrm{~b}$ represents two repeat AGC profiles for the same track: the pattern is very reproducible within $0.3 \mathrm{~dB}$. It shows a slow decrease of about $2 \mathrm{~dB}$ along the arc, with variations on a superimposed $15 \mathrm{~km}$ wavelength. These AGC undulations, being reproducible, are probably geophysically significant. At the end of the track, because of an abrupt change in slope, the control loops failed to maintain the signal within the window and the track was interrupted.

We further investigated the medium-scale signals by doing a spectral analysis over a longer profile. Figure 6a shows an altimetric height profile for a $300 \mathrm{~km}$ long path. As in Figure $5 \mathrm{a}$, the residues relative to a parabolic profile (Fig. 6b) show regular medium-scale undulations, of wavelength $20 \mathrm{~km}$ and height amplitude about $5 \mathrm{~m}$. This is clearly visible in the spectral analysis (Fig. 6c). The same analysis has been done for AGC, along the same track. The pattern shows a large-scale signal varying from 24 to $28 \mathrm{~dB}$ (Fig. 6d). When this large-scale trend is removed, residues of $1 \mathrm{~dB}$ amplitude appear (Fig. 6e), and the spectral analysis (Fig. 6f) shows a $10 \mathrm{~km}$ wavelength signal. These mediumscale variations of AGC are probably related to the medium-scale topography. We will explain latter why the wavelength of AGC variations is half that of the topography. 

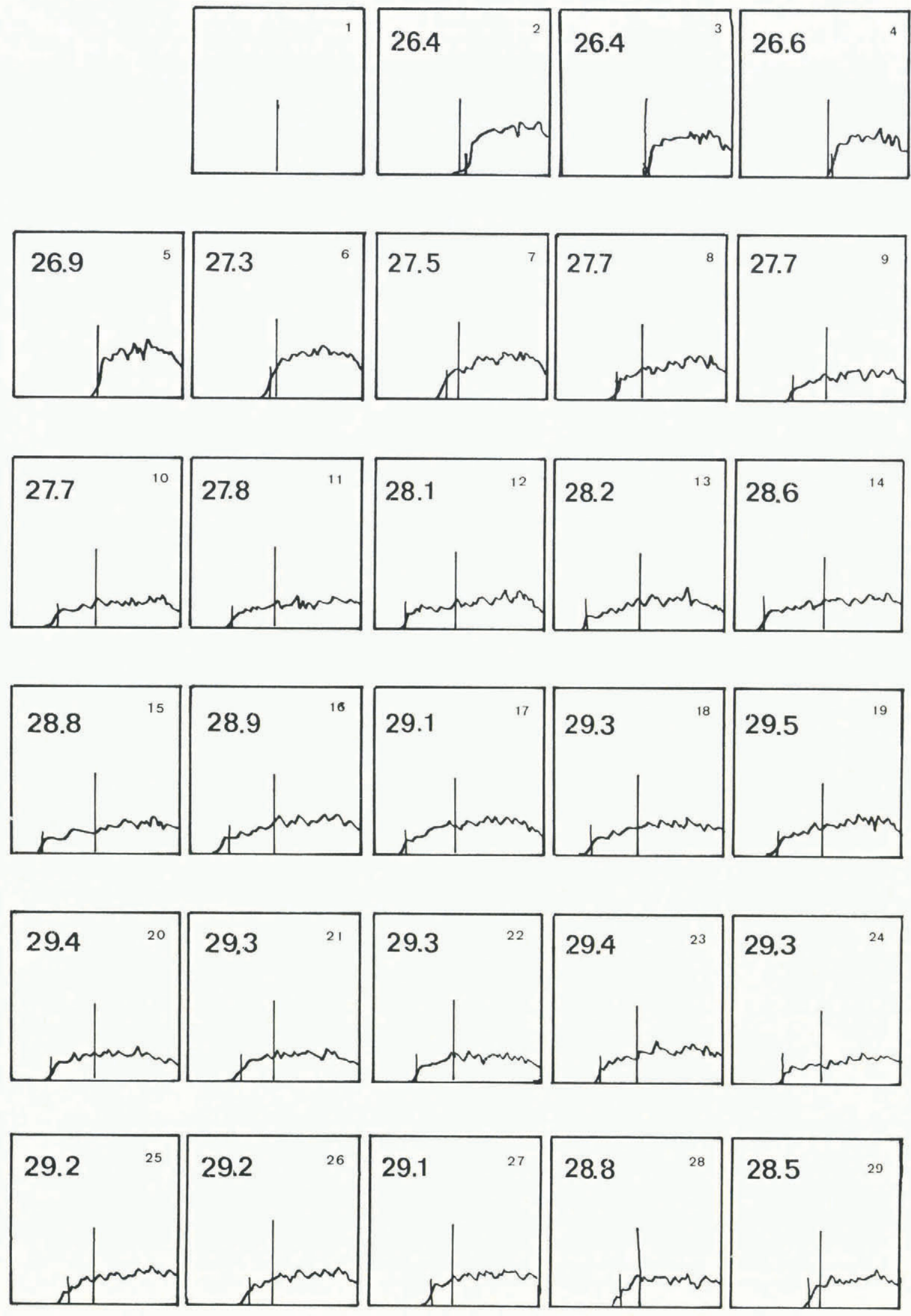

Fig. 2. Sequence of $0.1 \mathrm{~s}$ averages of the return wave form above Antarctica showing the relation between the AGC and the tracker error. The AGC value is given in the upper left corners of the diagrams, expressed in decibels. 


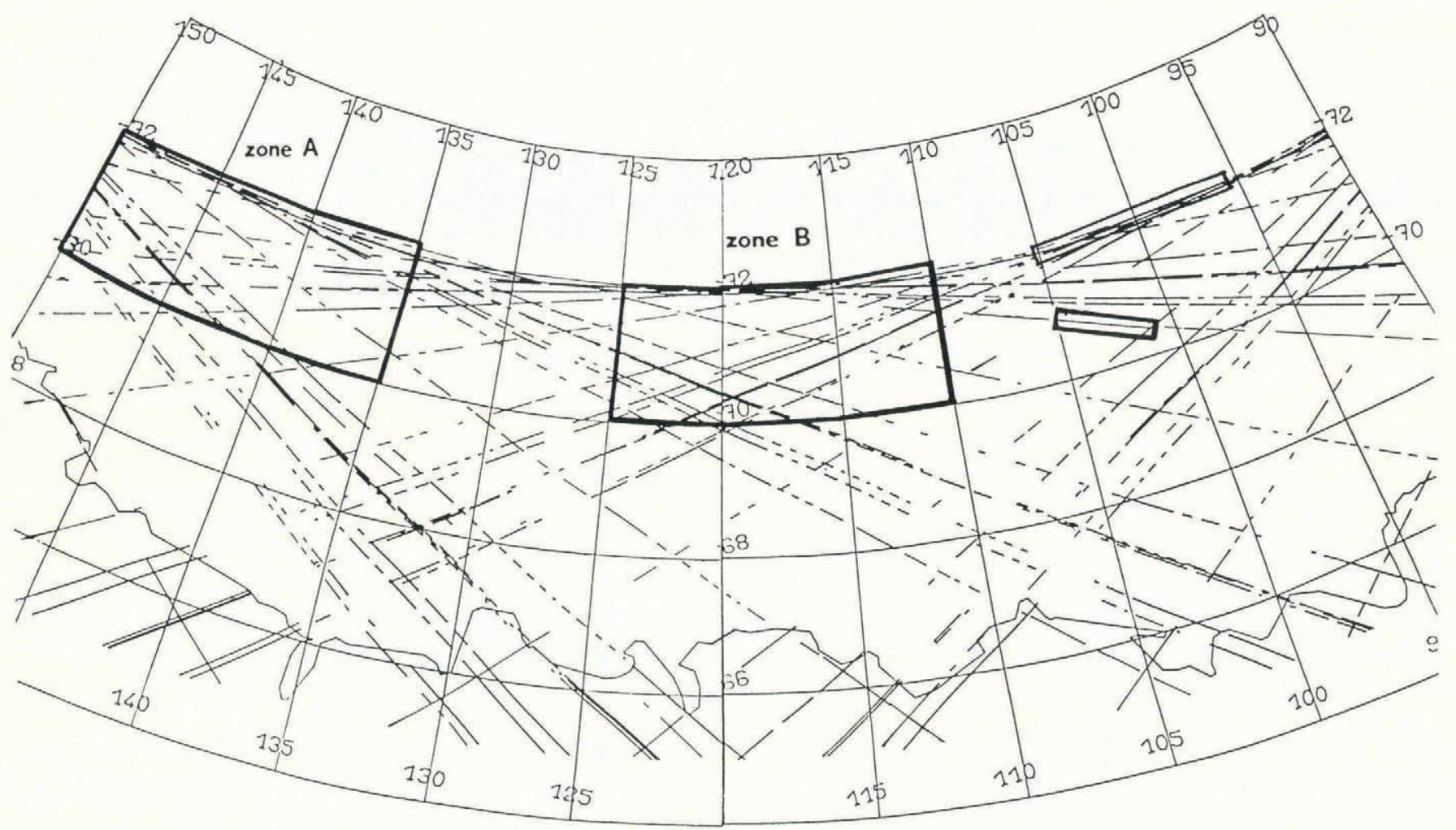

Fig. 3. Map of the Seasat altimeter data used in the present analysis. The profiles shown in Figures 5 and 6 , and the two zones $A$ and $B$ compared in Figure 8, are also indicated.

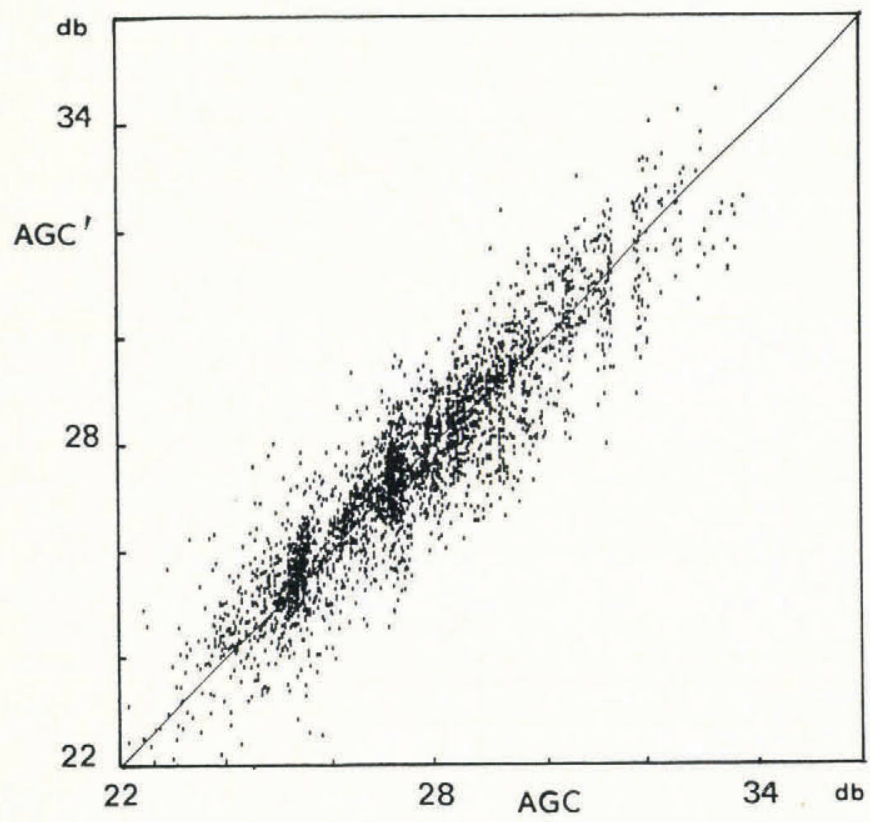

Fig. 4. Plot of the corrected Automatic Gain Control ( $A G C^{\prime}$ ) versus the measured AGC. The r.m.s. difference is I db. The gap near $32 d B$ is probably due to malfunctioning of the instrument.
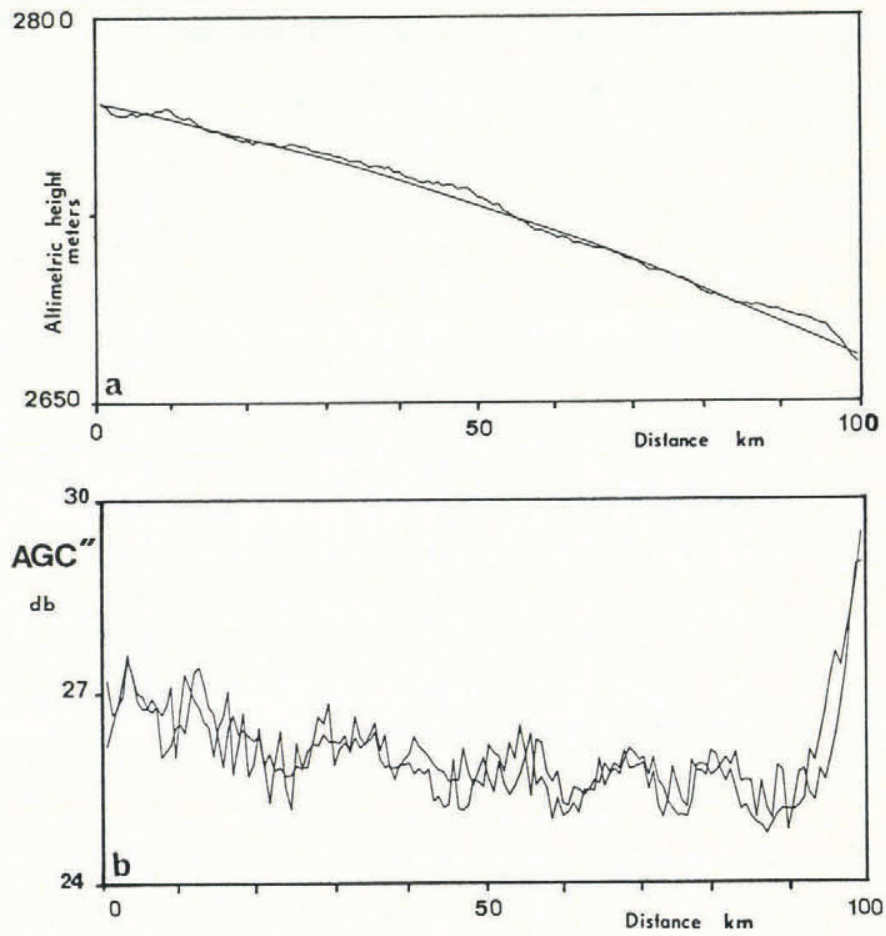

Fig. 5.(a) Retracked altimetric heights along a $100 \mathrm{~km}$ long path of the repetitive orbit of Seasat. The smooth curve is a parabolic fit along the profile. (b) Corresponding profiles for $A G C$. 

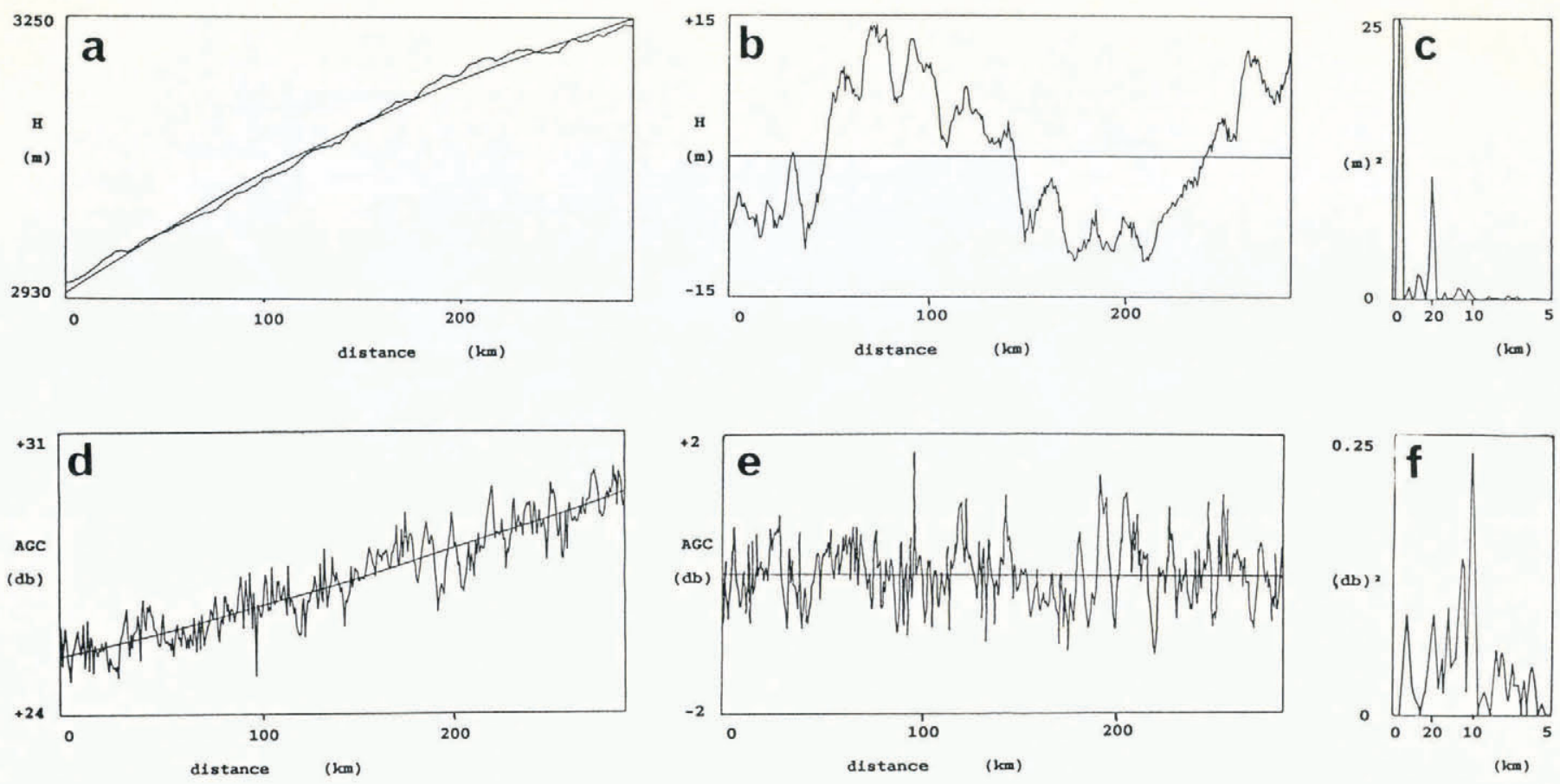

Fig. 6. (a) Retracked altimetric height along a $300 \mathrm{~km}$ long path, with a parabolic fit superimposed. (b) Residues between altimetric height and the parabolic fit. (c) Spectral analysis of the residues. Note the $20 \mathrm{~km}$ wavelength undulations. (d), (e), and (f) represent the same treatment for $A G C^{\prime}$, for the same path. Note the $10 \mathrm{~km}$ wavelength undulations.

\subsection{Large-scale AGC observations}

The overall variations of AGC for the entire area are of the order of $15 \mathrm{~dB}$ around a mean value of $27 \mathrm{~dB}$ (Fig. 7). Though the distribution of these values is rather Gaussian, systematic large-scale geographical variations can be observed. Figure 8 represents the distribution of values for two domains, shown in Figure 3. The scatter in each domain is about half that on the large scale: the mean

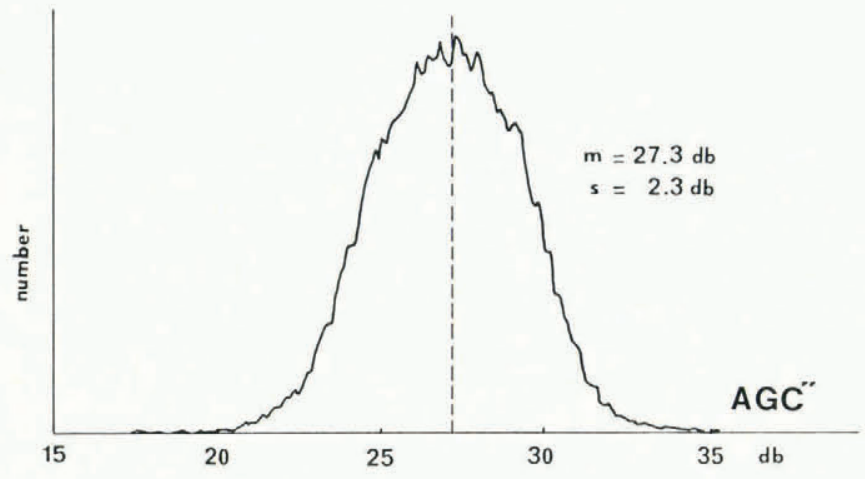

Fig. 7. Distribution of the corrected Automatic Gain Control $A G C^{\prime} . m$ is the average and $s$ the root mean squares.

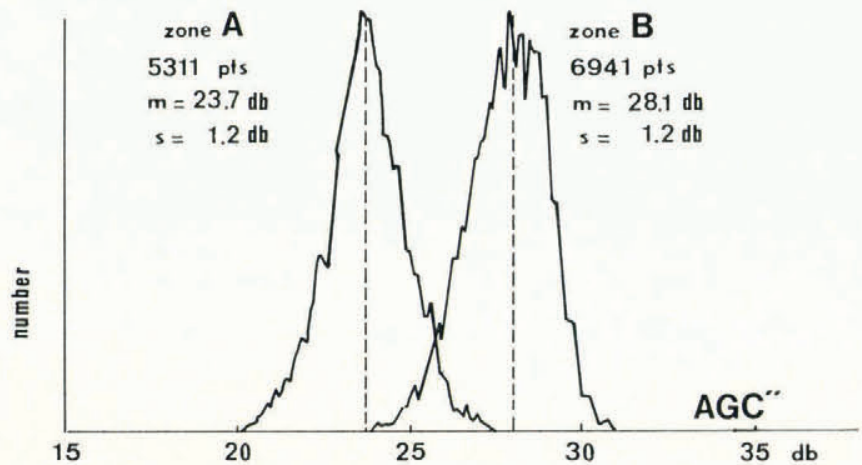

Fig. 8. Distribution of $A G C$ in two domains $A$ and $B$, represented in Figure 3. values in each domain are significantly different, of the order of 24 and $28 \mathrm{~dB}$, respectively.

Figure 9 is a map of AGC values. The data have been averaged in $20 \times 30 \mathrm{~km}^{2}$ domains, the number of data points in each domain being of the order of 25 . The medium-scale signal must vanish after the averaging process. The values vary from 20 to $35 \mathrm{~dB}$. The variations are very smooth. Neither the slope nor the altitude of the superimposed topography seems to be related to the signal. Partington and others (1989) have already mentioned that no correlation was found between wave form and altitude. If volume scattering is dominant, one would expect a correlation between wave form (or $\mathrm{AGC}$ ) and temperature (or altitude).

In Figure 10, the katabatic wind flow lines (Parish, 1982) of this area are superimposed on the $\langle A G C\rangle$ map. These winds are stronger where the topography channels them. The case for intense winds near Cape Denison (along long. $140^{\circ} \mathrm{E}$.) is particularly well known (Parish, 1982). By eye, it seems that 〈AGC〉 is smaller (of the order of $20 \mathrm{~dB}$ ) in areas of wind chanelling (near long. $100^{\circ}$ and $140^{\circ} \mathrm{E}$.) and larger (near $30 \mathrm{~dB}$ ) in areas of domes and slower wind speeds (near zone B in Figure 3). Note that, although katabatic wind intensity is related to topography, the observed correlation between AGC and wind cannot be explained by an effect of topography on AGC. In particular, the areas where strong katabatic winds are found are concave on a scale larger than $200 \mathrm{~km}$. The altimetric return is sensitive to concavity and slope on the scale of the footprint, of the order of $10 \mathrm{~km}$.

For quantifying this comparison, Parish's flow line has been digitized and the wind assumed to be inversely dependent on the flow-line distance. In other words, the flux is assumed to be constant between two flow lines, as defined by Parish (1982). In Figure 11, this wind intensity is plotted against the $\langle A G C\rangle$ values binned every dB. Seven hundred regions are used. The linear correlation coefficient is 0.56, which is significant at more than the $99 \%$ confidence level. We therefore find evidence that the corrected Automatic Gain Control, averaged on a medium scale, is mostly sensitive to wind-induced effects.

In order to support a quantitative comparison, one can scale the wind intensity: the wind measurement at Pioneerskaya Station (lat. $69.7^{\circ} \mathrm{S}$., long. $95.5^{\circ} \mathrm{E}$.) and at Charcot Station (lat. $69^{\circ} 05^{\prime} \mathrm{S}$., long. $138.5^{\circ} \mathrm{E}$.) is $10 \mathrm{~m} / \mathrm{s}$ (Kodama and Wendler, 1986); the corresponding AGCs for both stations are $25 \mathrm{~dB}$. This scaling leads to an average 

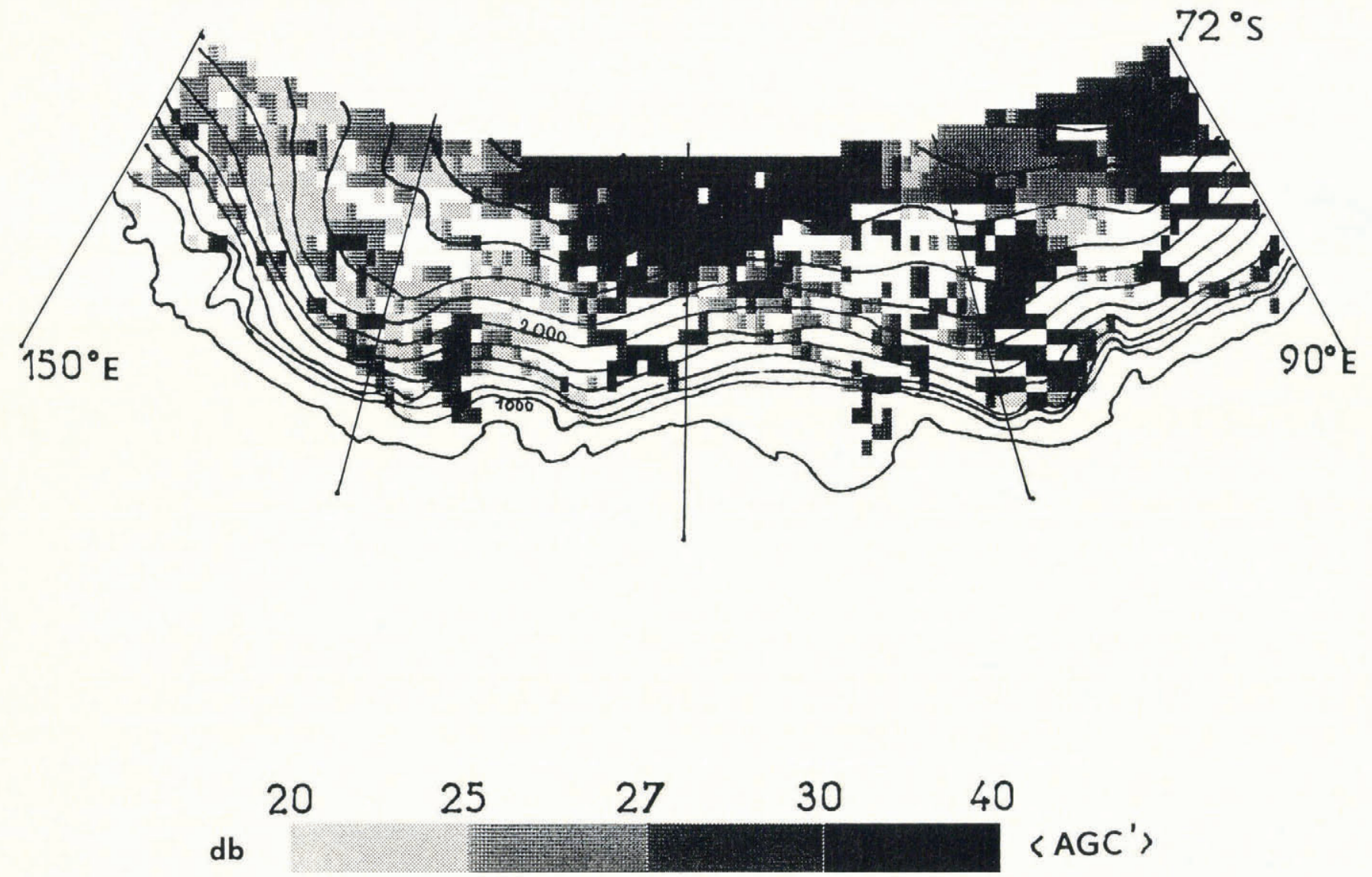

Fig. 9. Map of $\left\langle A G C^{\prime}\right\rangle$ averaged over $20 \times 30 \mathrm{~km}^{2}$ domains. The topographic contours are from Drewry (1983).

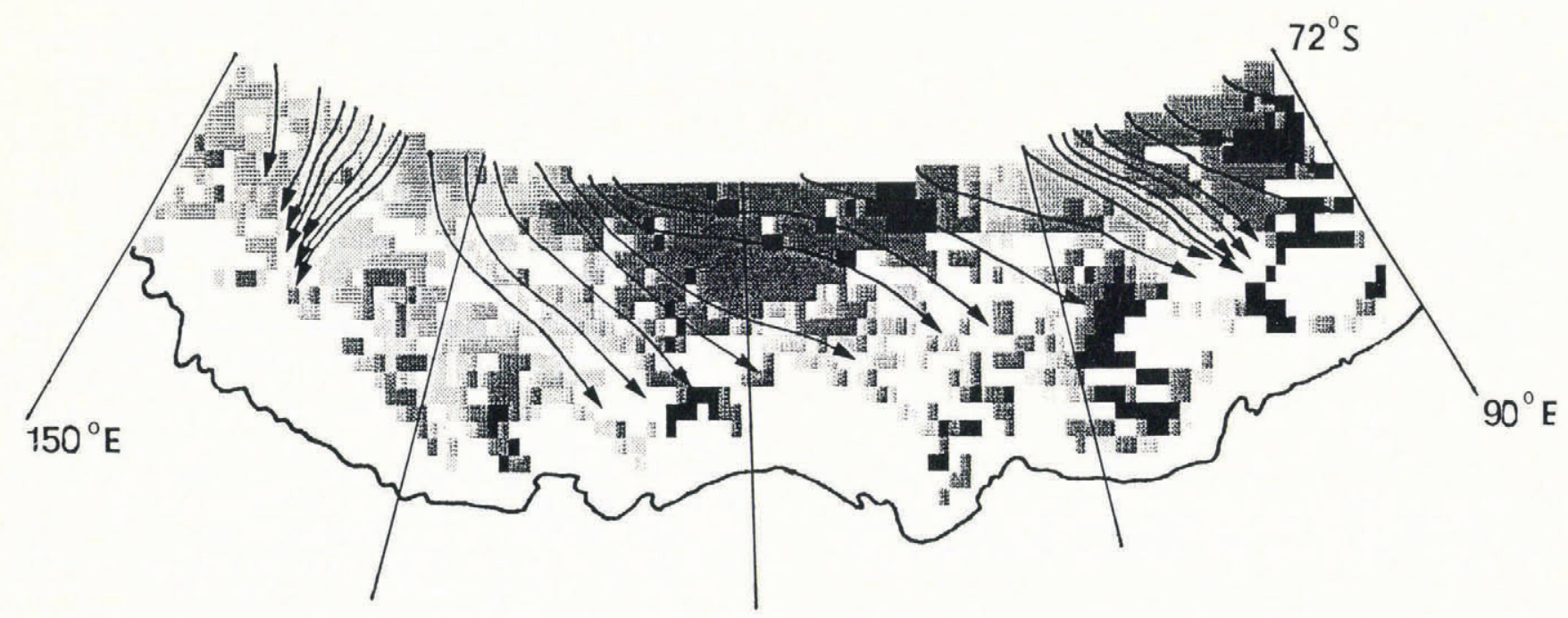

$\begin{array}{cccccc}\mathrm{db} & 20 & 25 & 27 & 30 & 40 \\ 10^{-3} & 16 & 5.3 & 3.3 & 1.6 & .16 \quad\left\langle\mathrm{~s}^{2} \text { > }\right.\end{array}$

Fig. 10. Same as Figure 9 with katabatic flow lines of Parish (1982) superimposed. The values are also scaled in quadratic mean slope of the surface as explained in the text. 


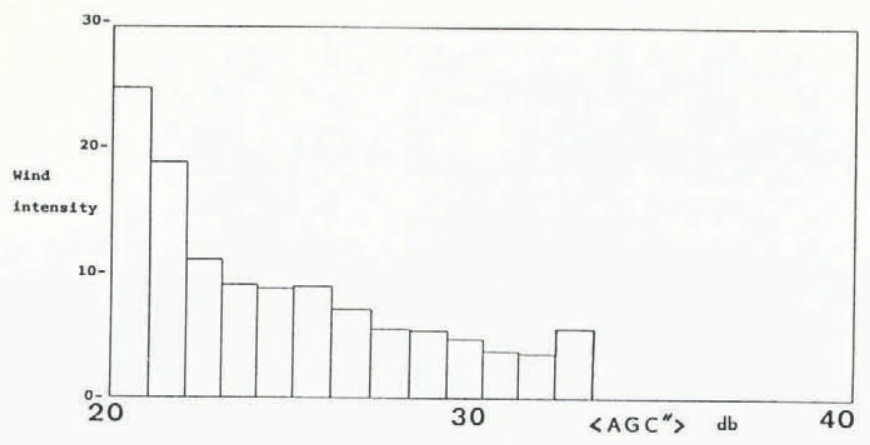

Fig. 11. Relation between $A G C$ and wind intensity deduced from Parish (1982) and averaged for each $1 \mathrm{~dB}$ interval of $\langle A G C\rangle$.

wind intensity in the sector of $7.5 \mathrm{~m} / \mathrm{s}$, corresponding to the average AGC value of $27 \mathrm{~dB}$ (Fig. 7). The estimated wind intensity varies between 5 and $25 \mathrm{~m} / \mathrm{s}$, which is consistent with in-situ observations.

One could $a$ priori imagine using these data to provide a map of katabatic wind intensity above Antarctica, and its possible seasonal variations. An approach, not used further here, would be to use the empirical relation between 〈AGC〉 and wind as given in Figure 11. This would integrate all possible physical effects on AGC. Note that this approach would not be different from that used for estimating altimetric winds above the ocean (Chelton and Wentz, 1986). As this relation is apparently not linear, for a given precision of $\langle A G C\rangle$ the sensitivity of the wind determination will be smaller for strong winds than for light winds. This is similar to the case for the ocean.

To understand the physical control of the altimetric return intensity, we now model AGC. The strong observed variations of AGC correspond to variations of the total energy, by a factor 30: a parameter able to provide such a variation must be easily detected after a theoretical analysis.

\section{SURFACE SCATTERING}

\subsection{The analytical formulation for AGC}

The theoretical model for the wave forms and for their integral will depend on the nature of the scattering. For a surface echo, the Brown (1977) model can be used to describe altimeter wave forms. The elements of this model are briefly recalled. For a flat surface, the average return power $P_{\mathrm{r}}(t)$ as a function of time $t$ can be written as

$$
P_{\mathrm{r}}(t) \mathrm{d} t=P_{\mathrm{r}}(0) \exp (-a t) I_{0}\left(b t^{\frac{1}{2}}\right) \mathrm{d} t .
$$

The origin of the time corresponds to the middle of the leading edge of the wave form. $a$ and $b$ which depend on the angle $\theta$, between the pointing direction and the surface impact direction, are expressed in Table $I_{0} I_{0}$ is the Bessel function of the second kind, and $P_{\mathrm{r}}(0)$ can be expressed by

$$
P_{\mathrm{r}}(0)=K \sigma_{0} \exp \left(-4 / G \sin ^{2} \theta\right)
$$

where $G$ is the antenna gain (see Table I).

\section{TABLE I. DEFINITION OF THE SEASAT ALTIMETER} PARAMETERS
Definition

Value

\section{$(4 / c) G H \cos (2 \theta)$}

$4 / G(c H)^{\frac{1}{2}} \sin (2 \theta)$

Velocity speed

Antenna gain

Satellite range

\section{$\approx 0.008$}

$\approx 0.262 \theta$

$3 \times 10^{8} \mathrm{~m} / \mathrm{s}$

$5.6 \times 10^{-4}$

$800 \mathrm{~km}$ $\sigma_{0}$ is the back-scatter cross-section per unit area and $K$ is the system constant defined in McArthur (1978):

$$
K=\left(P_{\mathrm{t}} G^{2} \lambda^{2}\right) /\left(64 \pi^{2} H^{3}\right),
$$

$P_{\mathrm{t}}$ being the transmitted power, $\lambda$ the radar wavelength, and $H$ the satellite range.

If a Gaussian surface roughness is added, the average return is then a convolution of the height probability density function of the specular points with $P_{\mathrm{r}}(t)$, which expresses that some part of the signal will come back earlier. One can easily verify that the total energy or the integral of $P_{\mathrm{r}}(t)$ is preserved.

The total received energy is then the integral of Equation (4) during the whole window aperture. After replacing $a$ and $b$ by their values (Table I) and developing the Bessel function, one can show that

$$
\int_{-T}^{T} P_{\mathrm{r}}(t) \mathrm{d} t=\left(53.34+13.6 \theta^{2}\right) P_{\mathrm{r}}(0)
$$

where $2 T$ is the duration of the receiving window, $60 \times 3.125 \mathrm{~ns}$. Note that this equation is similar to the on-board tracking equation for $\theta^{2}=0$. If not, retracking slightly underestimates the altimetric height. One can now write AGC with respect to the physical parameters:

$$
\mathrm{AGC}=K_{\mathrm{dB}}+\sigma_{\mathrm{dB}}+A \theta^{2} .
$$

For Seasat, the nominal value of $K_{\mathrm{dB}}$ is $20 \mathrm{~dB}$, but a larger value $(22 \mathrm{~dB})$ seems to be required (Chelton and McCabe, 1985). $A$ is equal to 8.4 if $\theta$ is expressed in degrees.

\subsection{The effects of the parameters}

a. The surface-slope effect

The term $A \theta^{2}$ is generally named the attitude loss, because for a flat surface like the ocean, $\theta$ is only due to the antenna pointing angle, $p$. $p$ varied from $0^{\circ}$ to $0.53^{\circ}$ for Seasat and was corrected for through an on-board algorithm. Here, the incidence angle is also dependent on the surface slope $i$, on the altimeter footprint scale (Fig. 12). In principle, one should also consider the angle due to the Earth's ellipticity, about $0.15^{\circ}$ at the Antarctica latitudes.

The incidence angle is related to $i$ and $p$ by:

$$
\cos \theta=\cos p \cos i+\cos \Omega \sin p \sin \theta \text {. }
$$
write:

As these angles, except $\Omega$, are very small, one can

$$
\theta^{2}=p^{2}+i^{2}+2 p i \cos \Omega .
$$

The variations in $p$ are very slow, and can be seen as a constant bias on a profile scale. Drewry and others (1985) have provided model continental ice-surface topographies. They first distinguish large-scale features which are described by nearly parabolic dependence of height upon distance; the induced slope is around $0.06^{\circ}$ for an average ice-sheet altitude, and vary on a very large scale. On the contrary, the medium-scale features are typically represented by isotropic "bell-shaped mounds" of $10 \mathrm{~m}$ elevation and $20 \mathrm{~km}$ size; the induced slope varies by more than $0.2^{\circ}$, on a wavelength half that of the topography (the slope vanishes at the bottoms and at the tops of the undulations, and reaches extremes on both flanks).

The total effect, for such values, is a white noise of about 1 or $2 \mathrm{~dB}$ amplitude on $\mathrm{AGC}$, and a signal at the half-undulation wavelength of about $0.3 \mathrm{~dB}$ (for the given example). The profile shown in Figure 6 belongs to a typical Antarctic region (see Remy and others, 1989). The $10 \mathrm{~km}$ wavelength signal identified by the spectral analysis of AGC is probably due to the $20 \mathrm{~km}$ wavelength undulation signal on the topography. The observed amplitude of $0.23 \mathrm{~dB}$ seems to be consistent with the topography.

\section{b. The back-scatter coefficient effect}

In the simplest approximations, the back-scatter coefficient for diffuse reflection above a rough surface is (e.g. Barrick, 1968; Rott, 1984): 


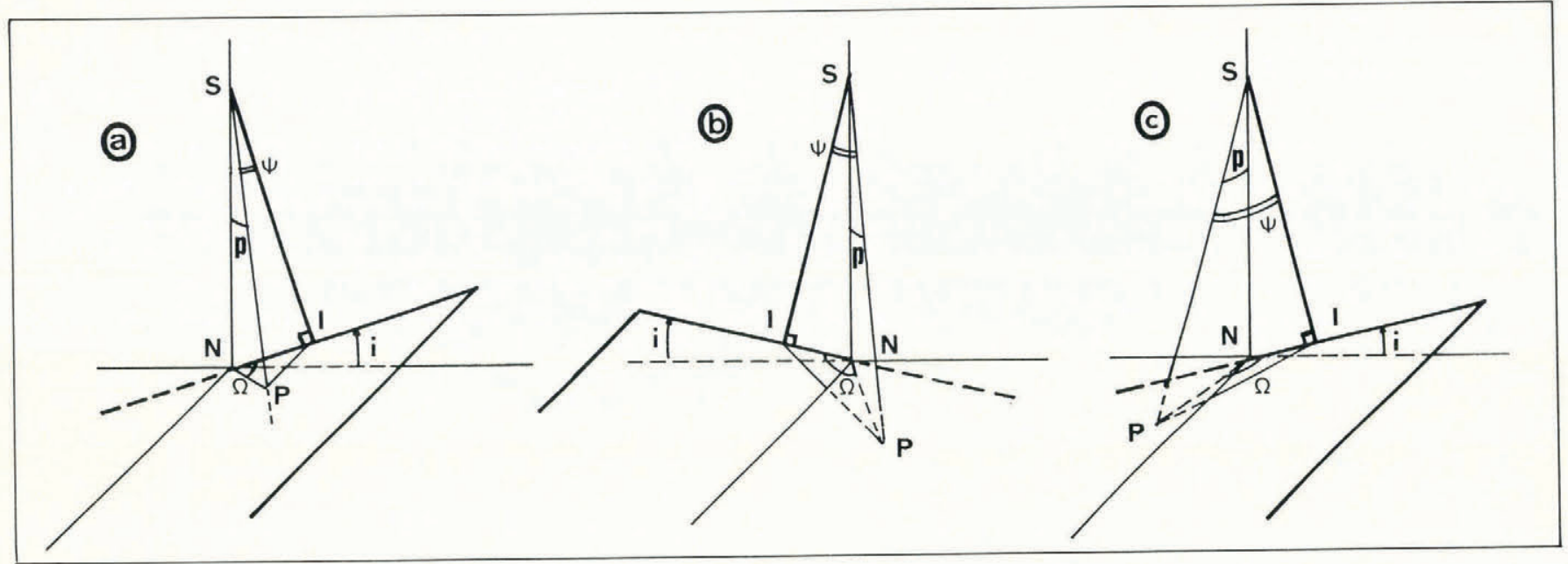

Fig. 12. Geometry of the problem: $p$ is the pointing angle of the antenna relative to the nadir direction, $i$ is the slope of the surface, and $\theta$ is the incidence angle. $\Omega$ can take any value in $a$ given area, depending on the variation in local slope (b) or for different passes with different pointing or azimuth angles (c).

$$
\sigma_{0}=\frac{1}{2} R^{2} / S^{2}
$$

where $S^{2}$ is the quadratic mean slope of the surface on the radar wavelength scale, and $R^{2}$ is the Fresnel reflection coefficient at normal incidence which depends on the dielectric constant:

$$
R=\left((\varepsilon)^{\frac{1}{2}}-1\right) /\left((\varepsilon)^{\frac{1}{2}}+1\right) .
$$

For dry snow, $\varepsilon$ in turn depends only on the snow density $\rho$ (Tiuri and others, 1984):

$$
\varepsilon=1+1.7 \rho+0.7 \rho^{2} .
$$

As the surface-snow density on continental ice varies from 0.35 to 0.45 , we will assume for $R$ a constant value of 0.145 .

\subsection{Geophysical interpretation}

We will show that the medium-scale variations of AGC can be due to slope effect (Equations (8) and (10)), and find evidence that the large-scale variations can be related to $S^{2}$ variations.

On the wavelengths of significance for scattering of the radar signal, the literature describes micro-roughness of wavelength $30 \mathrm{~cm}$ and height varying from 0.5 to $2 \mathrm{~cm}$ (e.g. Poggi, 1977; Fung and Eom, 1982). The small-scale roughness, sastrugi, and snow dunes having wavelengths of 6-30 $\mathrm{m}$ and heights of $0.5-2 \mathrm{~m}$ (Drewry and others, 1985), can play a marginal role in $S^{2}$.

These small-scale features vary with wind intensity and are related to the surface-roughness parameter used in micrometeorological descriptions of the atmospheric boundary layer (e.g. Holmgren, 1971; Poggi and others, 1982). As explained by Poggi (1977), for surfaces affected by asperities of constant height, the surface roughness is proportional to this height. The constant of proportionality is about 10, based on the wind-tunnel experiments of Lettau (1971). The values given by Paterson (1981) vary between $0.1 \mathrm{~mm}$ for new snow to $11 \mathrm{~mm}$ for coarse snow with sastrugi. Using Gaussian distribution for microroughness, one can calculate that, through $S^{2}$ in Equation (11), variations in the back-scatter coefficient due to these features are up to $40 \mathrm{~dB}$.

As an example, we have parameterized the $\langle A G C\rangle$ values in terms of the surface roughness as used by micrometeorologists. By neglecting the $A \theta^{2}$ term, one can deduce $S^{2}$ from $\langle\mathrm{AGC}\rangle$. Its values vary from $10^{-2}$ to $10^{-4}$. Assuming that the micro-roughness correlation length is $30 \mathrm{~cm}$, this provides the micro-roughness amplitude which varies from 2.7 to $0.5 \mathrm{~cm}$. This is equivalent to a roughness parameter of $2.7-0.5 \mathrm{~mm}$, for an 〈AGC〉 varying from 20 to $35 \mathrm{~dB}$. These values are in good agreement with those derived from the micrometeorological measurements (Paterson, 1981).

\section{VOLUME SCATTERING}

\subsection{The analytical formulation}

For the volume scattering, we will use the Ridley and Partington (1988) model, modified by Partington and others (1989) to account for the difference between propagation speeds of radiation in snow and air. Some assumptions are required: the snow-pack must be homogeneous within the altimeter footprint, ice grains must be spherical, and only single scattering is considered. The latter assumption may drastically overestimate volume scattering.

The power received at time $t$ is as follows:

$$
P_{\mathrm{r}}(t) \mathrm{d} t=M\left(1-\exp \left(-2 k_{\mathrm{e}} c t / n\right)\right) /(H+c t / n)^{2} \mathrm{~d} t
$$

where the time origin, $t=0$, corresponds to the beginning of the leading edge of the wave form. $n$ is the refractive index of snow, taken as 1.25 ( $n$ is the inverse of the root square of the dielectric constant given in Equation (13)); $k_{\mathrm{e}}$, the extinction coefficient, is the sum of the absorption coefficient $k_{\mathrm{a}}$ and the scattering coefficient $k_{\mathrm{s}} . M$ is defined as follows:

$$
M=6 \pi T / 8 K H^{3}\left(1-\exp \left(-k_{\mathrm{s}} c \tau / n\right)\right)
$$

where $K$ is the system constant defined in Equation (6), $\tau$ is the gate duration $(3.125 \mathrm{~ns})$, and $T$ is the surfacetransmission coefficient; it is linked with the Fresnel coefficient, and is close to 1 .

Then, the total received energy per unit area (normalized by $\pi H c \tau$ ), is given by integration of Equation (14). By neglecting $c t / n$ compared to $H$ :

$$
\begin{aligned}
& \int_{-T}^{T} P_{\mathrm{r}}(t) \mathrm{d} t= \\
& =K^{\prime}\left(1-\exp \left(-k_{\mathrm{s}} c \tau / n\right)\right)\left[x_{\mathrm{m}}+\left(\exp \left(-2 k_{\mathrm{e}} x_{\mathrm{m}}\right)-1\right) /\left(2 k_{\mathrm{e}}\right)\right]
\end{aligned}
$$

where

$$
K^{\prime}=6 n K T /(8 c \tau) \text {. }
$$

Expressed in decibels, $K^{\prime}$ is equal to $25 \mathrm{~dB}$, if $K$ equals $22 \mathrm{~dB} . x_{\mathrm{m}}$ is the vertical maximum depth reached by the radar wave. If the radar range in dry snow is equal to $37 \mathrm{~cm}, x_{\mathrm{m}}$ is then equal to $11 \mathrm{~m}$.

\subsection{Geophysical interpretation}

As already mentioned, the small variations in the dielectric constant (Equation (13)) lead to small variations in 


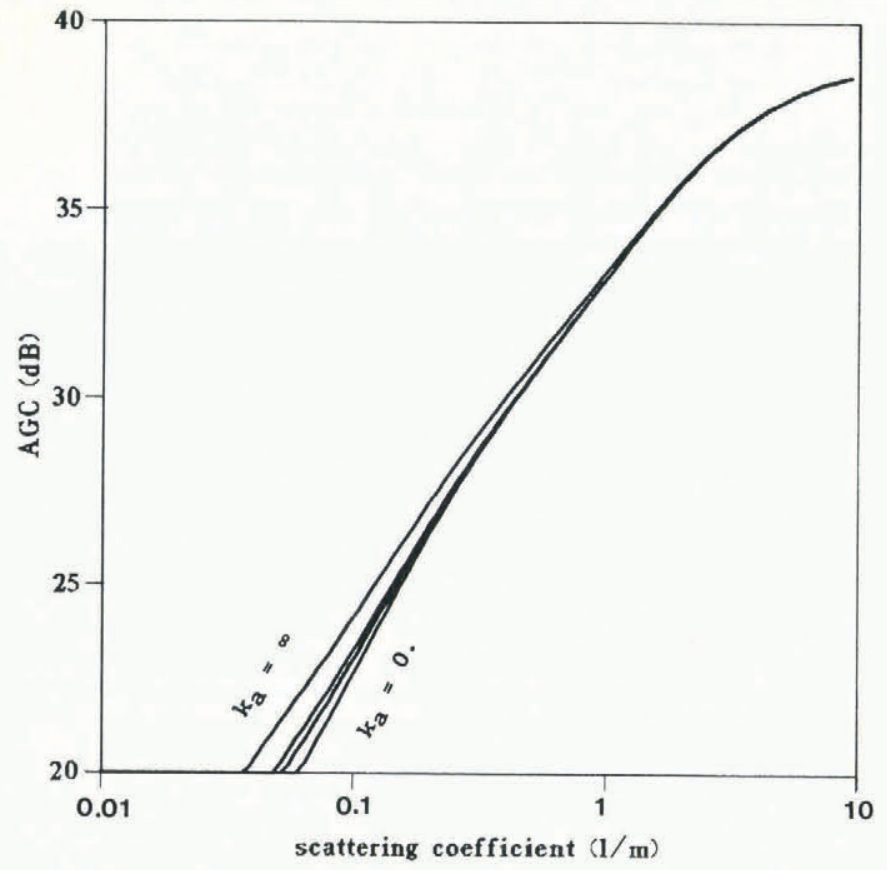

Fig. 13. In the hypothesis of volume scattering, AGC versus scattering coefficient, for different values of the absorption coefficient. Note that one can neglect variations in this latter parameter.

the refractive index or in the transmission coefficient. The uncertainty in the actual behaviour of $k_{\mathrm{a}}$ and $k_{\mathrm{s}}$ is a serious limitation for the modelling of volume scattering. Yet, in Figure 13, Equation (16) is computed for $k_{\mathrm{a}}$ varying from 0 to $\infty$, and $k_{\mathrm{s}}$ from 0.01 to $10 \mathrm{~m}^{-1}$. The variation in the absorption coefficient does not affect the total intensity. Only variations in the scattering coefficient can explain AGC variations. The scattering coefficient is related to snow density and to grain volume: to describe the $15 \mathrm{~dB}$ observed $\mathrm{AGC}$ signal, variations in $\mathrm{k}_{\mathrm{S}}$ by a factor of 30 are required.

Such variations are supported, neither by direct measurements of the scattering coefficient, as provided by passive microwave observations, nor by direct measurements of the snow parameters.

Rotman and others (1982) used the properties of the scattering coefficient in the microwave domain to deduce the accumulation rate from passive radiometer observations. They assumed that the accumulation rate is inversely proportional to the snow-grain volume or to the scattering coefficient as measured by passive radiometer observations (Zwally, 1977). They derived accumulation rates which are consistent with in-situ data within a factor of 2 . If the scattering coefficient is sufficiently affected by the wind for explaining the wind versus AGC correlation, this would have a dramatic result: the wind pattern would be directly visible on the map for accumulation rate as deduced from passive radiometer observations, which is not the case.

For obtaining an order of magnitude on the expected variations of the snow-grain volume, we will choose the equation given by Comiso and others (1982), and neglect the density dependence.

$$
k_{\mathrm{s}}=0.35 d^{3}
$$

where the grain-size is in $\mathrm{mm}$. The order of magnitude of the AGC variations could be explained if $k_{\mathrm{s}}$ varies from 0.05 to $10 \mathrm{~m}^{-1}$, or grain-size varies from about 0.5 to $2 \mathrm{~mm}$. In this hypothesis, strong winds would induce a grain-size of about $0.5 \mathrm{~mm}$, and a grain-size larger than $2 \mathrm{~mm}$ would be found in regions with low wind intensity. Indeed, Colbeck (1980) mentioned that wind may break the snow grains, but sparse measurements in Antarctica, at Byrd Station or at Plateau Station (Gow, 1969) give values between 0.3 and $0.5 \mathrm{~mm}$ for the first meter. This would, in both cases, correspond to low values of AGC. Moreover, Narita and Maeno (1979), by studying grain-size at Mizuho Station where katabatic winds are very strong, found a

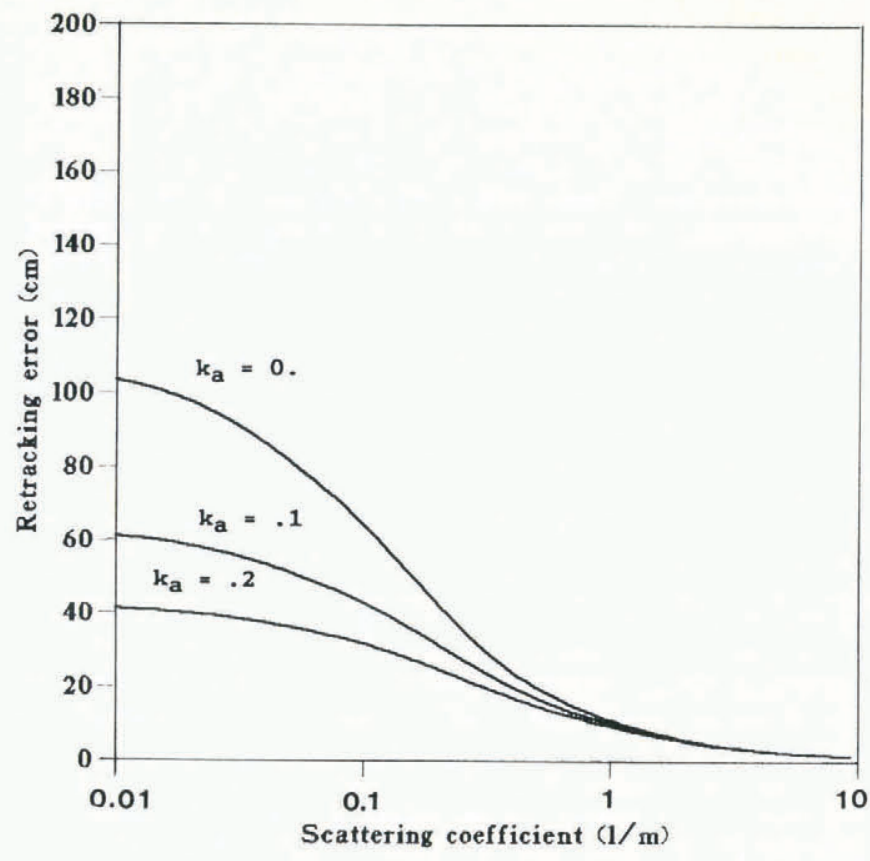

Fig. 14. Altimetric height error induced by the retracking method used, in the case of pure volume scattering, versus scattering coefficient and for different values of the absorption coefficient. Note that the error is within $a$ meter.

value $(0.56 \mathrm{~mm})$ similar to that at Plateau Station, where the wind intensity is low. Up to now, no measurement in the literature points to such large variations in grain-size, on the large scale.

Furthermore, volume scattering cannot explain the AGC variations at the half-wavelength of the medium-scale surface undulations (Fig. 6): volume scattering does not depend on the incidence angle, above all near the vertical. Thus, the effect of the slope on volume scattering is highly unlikely.

Not only are such large observed variations supported with difficulty but also the model leads to low values of AGC, all the more as the single scattering hypothesis overestimates the estimation. However, when the wind is very strong, it is possible that surface scattering vanishes and the altimetric signal could be affected by volume scattering. Let us look at the induced height error on the retracked data.

\subsection{Induced error on the retracking technique}

By comparing Equation (14) with the total energy given in Equation (16) and dividing by 53, one can deduce the time $t$, or the corresponding height error induced by our retracking technique. The results are plotted in Figure 14 for different values of $k_{\mathrm{a}}$. First, one can demonstrate that the maximum retracking error, obtained for a zero absorption and a zero scattering is less than $1.37 \mathrm{~m}$. Secondly, for a significant scattering $\left(k_{\mathrm{S}}>0.2\right.$, or $\mathrm{AGC}>$ 27 , i.e. half of the data), the maximum induced error is reached for $k_{\mathrm{a}}=0$ and is of the order of $40 \mathrm{~cm}$.

In conclusion, the larger the scattering coefficient, the more the signal comes from the upper snow layer. This is an important remark, because it provides a means for selecting the region where volume scattering does not affect the altimetric surface measurement significantly. Remy and others (1989) showed that above Antarctic lakes, where the surface-slope error vanishes, an inverse technique can provide a precision within $50 \mathrm{~cm}$. Then, if one selects regions with AGC greater than $30 \mathrm{~dB}$, one adds less than $10 \mathrm{~cm}$ residual error due to the volume-scattering effect.

\section{CONCLUSION}

We have analysed the processes which can affect the power return (or its Automatic Gain Control, AGC) of a radar altimeter above continental ice. On the medium scale, we observe variations of the order of few $d B$, which seem 
to be due to surface-slope variations. On the large scale, AGC is strongly correlated with katabatic wind intensity.

If surface scattering is dominant, the physical mechanism able to explain the correlation is the microroughness and the small-scale wind-induced features. As above the ocean, wind creates these features which decrease the intensity of the surface scattering. A simplified numerical application leads to a micro-roughness parameter, as used by the micrometeorologists, between 2.7 and $0.5 \mathrm{~mm}$, which is consistent with in-situ measurements Moreover, in this case, the observed correlation between medium-scale AGC variations and local surface slope can be explained theoretically.

In the case of volume scattering, the correlation with wind must be explained by induced variations in grainsize, via the scattering coefficient. The expected grain-size values vary from $0.5 \mathrm{~mm}$ for high wind regions to $2 \mathrm{~mm}$ for low wind regions. First, this is in contradiction to the observed variations in the scattering coefficient as deduced from passive radiometer observations. Secondly, such an important signal would undoubtedly have been reported in the literature. Also, the medium-scale variations cannot be explained.

We also show that the model values for volume scattering, even though overestimated by the single-scattering hypothesis, are too low to account for the signal when AGC is sufficiently strong. On the other hand, even in the case of pure volume scattering, the signal comes from the upper snow layer, when the intensity of the altimetric return is important. This provides a means for selecting regions for the survey of the ice-sheet topography by radar altimetry: when the AGC is large, the surface as seen by the radar is close to the actual one.

As above the oceans, the altimeter therefore has the potential for measuring the intensity of katabatic winds. In the case of the oceans, the sea surface responds quickly to variations in surface winds, so that the altimeter provides an estimate of the instantaneous wind. On the contrary, above ice sheets, it is expected that micro-roughness or grain-size rather relate to average wind intensities over some period of time. Further improvements in this analysis require observations of height-slope joint probability distribution of the ice surface and of grain-size, and their relations to wind. Such mapping will be achievable with the ERS-1 data over $80 \%$ of Antarctica and over all of the Greenland ice sheet. In addition, the instrument tracker of ERS-1 will follow the ice surface better, and it should be possible to estimate 〈AGC〉 with a precision better than $1 \mathrm{~dB}$.

\section{REFERENCES}

Barrick, D.E. 1968. Rough surface scattering based on the specular point theory. IEEE Trans. Antennas Propag., AP-16, 449-454.

Brooks, R.L., W.J. Campbell, R.O. Ramseier, H.R. Stanley, and H.J. Zwally. 1978. Ice sheet topography by satellite altimetry. Nature, 274(5671), 539-543.

Brown, G.S. 1977. The average impulse response of a rough surface and its application. IEEE Trans. Antennas Propag., AP-25, 67-73.

Chelton, D.B. and P.J. McCabe, 1985. A review of satellite altimeter measurement of sea surface wind speed: with a proposed new algorithm. J. Geophys. Res., 90(C3), 4707-4720.

Chelton, D.B. and F.J. Wentz. 1986. Further development of an improved altimeter wind speed algorithm. J. Geophys. Res., 91(C12), 14 250-14 260.

Colbeck, S.C., ed. 1980. Dynamics of snow and ice masses. New York, etc., Academic Press.

Comiso, J.C., H.J. Zwally, and J.L. Saba. 1982. Radiative transfer modeling of microwave emission and dependence on firn properties. Ann. Glaciol., 3, 54-58.

Drewry, D.J. 1983. The surface of the Antarctic ice sheet. In Drewry, D.J., ed. Antarctica: glaciological and geophysical folio. Cambridge, University of Cambridge. Scott Polar Research Institute, sheet 2.
Drewry, D.J., N.F. McIntyre, and P. Cooper. 1985. The Antarctic ice sheet: a surface model for satellite altimeter studies, In Woldenberg, M.J., ed. Models in geomorphology. Boston, etc., Allen and Unwin, 1-23.

Fung, A.K. and H.J. Eom. 1982. Application of a combined rough surface and volume scattering theory to sea ice and snow backscatter. IEEE Trans. Geosci. Remote Sensing, GE-20(4), 528-536.

Gow, A.J. 1969. On the rates of growth of grains and crystals in south polar firn. J. Glaciol., 8(53), 241-252.

Holmgren, B. 1971. Climate and energy exchange on a sub-polar ice cap in summer. Part C. On the katabatic winds over the north-west slope of the ice cap. Variations of the surface roughness. Uppsala, Uppsala Universitet. Meteorologiska Institutionen. (Meddelande 109.)

Kodama, Y. and G. Wendler. 1986. Wind and temperature regime along the slope of Adélie Land, Antarctica. $J$. Geophys. Res., 91(D6), 6735-6741.

Lettau, H. 1971. Antarctic atmosphere as a test tube for meteorological theories. In Quam, L.O., ed. Research in the Antarctic. Washington, DC, American Association for the Advancement of Science, 443-475. (Publication 93.)

McArthur, J.L. 1978. Seasat-A radar altimeter design description. Baltimore, MD, Johns Hopkins University. Applied Physics Laboratory. (Publication SDO-5232.)

McIntyre, N.F. and D.J. Drewry. 1984. Modelling ice-sheet surfaces for ERS-1's radar altimeter. ESA J., 8(4), 261-274.

Martin, T.V., H.J. Zwally, A.C. Brenner, and R.A. Bindschadler. 1983. Analysis and retracking of continental ice sheet radar altimeter waveforms. J. Geophys. Res., 88(C3), 1608-1616.

Mätzler, C. 1987. Applications of the interaction of microwaves with the natural snow cover. Remote Sensing Rev., 2, 259-388.

Narita, H. and N. Maeno. 1979. Growth rates of crystal grains in snow at Mizuho Station, Antarctica. Antarct. Rec., 67, 11-17.

Parish, T.R. 1982. Surface airflow over East Antarctica. Mon. Weather Rev., 110, 84-90.

Partington, K.C., J.K. Ridley, C.G. Rapley, and H.J. Zwally. 1989. Observations of the surface properties of the ice sheets by satellite radar altimetry. J. Glaciol., 35(120), 267-275.

Paterson, W.S.B. 1981. The physics of glaciers. Second edition. Oxford, etc., Pergamon Press.

Poggi, A. 1977. Introduction à la micrométéorologie. Transferts d'énergie atmosphère-sol. Paris, Masson.

Poggi. A., J.C. André, G. Wendler, D. Delaunay, D.J. Gosink, and Y. Kodoma. 1982. Interaction atmosphèreglace-océan en Antarctique. Météorologie, 29, 163-172.

Remy, F., P. Mazzega, S. Houry, C. Broussier, and J.F. Minster. 1989. Mapping of the topography of continental ice by inversion of satellite-altimeter data. $J$. Glaciol., 35(119), 98-107.

Ridley, J.K. and K.C. Partington. 1988. A model of satellite radar altimeter return from ice sheets. Int. J. Remote Sensing, 9(4), 601-624.

Rotman, S.R., A.D. Fisher, and D.H. Staelin. 1982. Inversion for physical characteristics of snow using passive radiometric observations. J. Glaciol., 28(98), 179-185.

Rott, H. 1984. Study on use and characteristics of SAR for land snow and ice applications. Paris, European Space Agency. (ESA Report 5441/83/D/IM(SC).)

Tiuri, M.E., A.H. Sihvola, E.G. Nyfors, and M.T. Hallikainen. 1984. The complex dielectric constant of snow at microwave frequencies. IEEE J. Oceanic Eng., OE-9377-382. Wingham, D.J., C.G. Rapley, and H.G. Griffiths. 1986. New techniques in satellite altimeter tracking systems. In IGARRS'86 Symposium, Zurich. Proceedings. Noordwijk, ESTEC. Scientific and Technical Publications Branch, 1339-1344. (ESA SP-254.)

Zwally, H.J. 1977. Microwave emissivity and accumulation rate of polar firn. J. Glaciol., 18(79), 195-215.

Zwally, H.J., R.A. Bindschadler, A.C. Brenner, T.V. Martin, and R.H. Thomas. 1983. Surface elevation contours of Greenland and Antarctic ice sheets. J. Geophys. Res., 88(C3), 1589-1596. 